\title{
MHD NATURAL CONVECTION FLOW ALONG A VERTICAL WAVY SURFACE IN PRESENCE OF HEAT GENERATION/ABSORPTION WITH VISCOSITY DEPENDENT ON TEMPERATURE
}

\author{
N. Parveen* and M. A. Alim \\ Department of Mathematics, Bangladesh University of Engineering and Technology, \\ Dhaka-1000, Bangladesh \\ *Corresponding email: nazma@math.buet.ac.bd
}

\begin{abstract}
The present numerical simulation is analyzed the Magnetohydrodynamic natural convection flow and heat transfer along a uniformly heated vertical wavy surface in presence of heat generation/absorption with temperature dependent variable viscosity. Using the appropriate transformations the governing boundary layer equations are reduced to non-dimensional forms. The resulting nonlinear system of partial differential equations are mapped into the domain of a vertical flat plate and then solved numerically applying implicit finite difference method together with Keller-box scheme. The solutions are expressed in terms of the skin friction coefficient, the rate of heat transfer, the streamlines as well as the isotherms over the whole boundary layer. The implications of heat generation/absorption parameter $(Q)$ and viscosity parameter $(\varepsilon)$ on the flow structure and heat transfer characteristics are investigated in detail while, Prandtl number (Pr), magnetic parameter $(M)$ and the amplitude-to-length ratio of the wavy surface $(\alpha)$ are considered fixed. Comparison with previously published work is performed and is found to be in good agreement.
\end{abstract}

Key Words: $M H D$, heat generation and absorption, temperature dependent viscosity, natural convection, uniform surface temperature, Keller-box method, wavy surface.

\section{INTRODUCTION}

The characteristics of natural convection flow of electrically conducting fluid in the presence of heat generation/absorption on magnetic field along a wavy surface is important from the technical point of view and such type of problems have received much attention of many researchers. If the surface is roughened the flow is disturbed by the surface and this alters the rate of heat transfer. Laminar natural convection flow from irregular surfaces can be used for transferring heat in several heat transfer devices, for examples, flat-plate solar collectors, flat-plate condensers in refrigerators, heat exchanger and geothermal reservoirs. The viscosity of the fluid to be proportional to a linear function of temperature, two semi-empirical formulae which was proposed by Charraudeau [1]. Yao [2-3] first investigated the natural convection heat transfer from an isothermal vertical wavy surface and used an extended Prantdl's transposition theorem and a finite-difference scheme. He proposed a simple transformation to study the natural convection heat transfer for an isothermal vertical sinusoidal surface. These simple coordinate transformations method to change the wavy surface into a flat plate. Transient free convection flow with temperature dependent viscosity in a fluid saturated porous media has shown by Mehta and Sood [4]. As per their investigation the flow characteristics substantially change when the effect of temperature dependent viscosity considered. Moulic and Yao [5] investigated natural convection along a wavy surface with uniform heat flux. The study of heat generation or absorption in moving fluids is important in problems dealing with chemical reactions and those concerned with dissociating fluids. Possible heat generation effects may alter the temperature distribution; consequently, the particle deposition rate in nuclear reactors, electronic chips and semiconductor wafers. In fact, the literature is replete with examples dealing with the heat transfer in laminar flow of viscous fluids. Vajravelu and Hadjinolaou [6] studied the heat transfer characteristics in the laminar boundary layer of a viscous fluid over a stretching sheet with viscous dissipation or frictional heating and internal heat generation. In this study they considered that the volumetric rate of heat generation, $q^{\prime \prime \prime}\left[W / m^{3}\right]$, should be $q^{m}=Q_{0}\left(T-T_{\infty}\right)$, for $T \geq T_{\infty}$ and equal to zero for $T<T_{\infty}$, where $Q_{0}$ is the heat generation/absorption constant. The above relation explained by Vajravelu and Hadjinolaou [6], is valid as an approximation of the state of some exothermic process and having $T_{\infty}$ as the onset temperature. When the inlet temperature are not less than $T_{\infty}$ they used $q^{m}=Q_{0}\left(T-T_{\infty}\right)$. The effect of temperature dependent viscosity on the free convective laminar boundary layer flow past a vertical isothermal flat plate in the region near the leading edge have been 
studied by Kafoussius and Williams [7]. Alam et al. [8] considered the problem of free convection from a wavy vertical surface in presence of a transverse magnetic field using Keller box method. They used a sinusoidal surface to elucidate the effects of magnetic field and the amplitude of the wavy surface on the velocity and temperature fields as well as on the local rate of heat transfer. They found that the effect of the magnetic parameter was to decrease the velocity profiles and to increase the temperature profiles and the amplitude of the sinusoidal surface results in decreasing the heat transfer rate. The combined effects of thermal and mass diffusion on the natural convection flow of a viscous incompressible fluid along a vertical wavy surface investigated by Hossain and Rees [9]. The effects of waviness of the surface on the heat and mass flux distributions in combination with the species concentration for a fluid having Prandtl number equal to 0.7 have been studied in that paper. The natural convection flow past a permeable wedge for the fluid having temperature dependent viscosity and thermal conductivity have been investigated by Hossain et al. [10]. Cheng [11] studied the natural convection heat and mass transfer near a vertical wavy surface with constant wall temperature and concentration in a porous medium. The problem of natural convection of fluid with temperature dependent viscosity along a heated vertical wavy surface have been studied by Hossain et al. [12]. Molla et al. [13] numerically investigated natural convection flow along a vertical wavy surface with uniform surface temperature in presence of heat generation/absorption. Mamun et al. [14] considered MHD-conjugate heat transfer analysis for a vertical flat plate in presence of viscous dissipation and heat generation. Molla et al. [15] also investigated natural convection laminar flow with temperature dependent viscosity and thermal conductivity along a vertical wavy surface. Numerical study on a vertical plate with variable viscosity and thermal conductivity has been investigated by Palani and Kim [16]. Very recently, Parveen and Alim [17] studied the effect of temperature dependent variable viscosity on magnetohydrodynamic natural convection flow along a vertical wavy surface. The above literature survey shows that heat generation/absorption effect on magnetic field with temperature dependent viscosity natural convection flow along wavy surface has not been well investigated.

In this current study the intensity of magnetic field natural convection flow along a vertical wavy surface in presence of heat generation/absorption with temperature dependent variable viscosity along a uniformly heated vertical wavy surface is studied. The boundary layer equations are reduced to dimensionless partial differential forms. The results are shown in terms of parametric presentations on the surface shear stress in terms of local skin friction and the rate of heat transfer in terms of local Nusselt number, the streamlines as well as the isotherms for various pertinent dimensionless parameters.

\section{MATHEMATICAL FORMULATION OF THE PROBLEM}

Consider the steady laminar two dimensional natural convection boundary layer flow of an electrically conducting viscous and incompressible fluid in presence of heat generation/absorption with variable viscosity along a vertical wavy surface. The surface temperature of the vertical wavy surface $T_{w}$ is uniform, which is greater than the ambient temperature $T_{\infty}$. A uniform magnetic field of strength $\beta_{0}$ is imposed along the $\bar{y}$-axis i.e. normal direction to the surface and $\bar{x}$-axis is taken along the surface. The boundary layer analysis outlined below allows $\bar{\sigma}(\bar{x})$ being arbitrary, but our detailed numerical work assumed that the surface exhibits sinusoidal deformations. The wavy surface may be described by

$\bar{y}_{w}=\bar{\sigma}(\bar{x})=\alpha \sin \left(\frac{n \pi \bar{x}}{L}\right)$

where $L$ is the wave length associated with the wavy surface.

The geometry of the wavy surface and the twodimensional cartesian coordinate system are shown in Fig. 1.

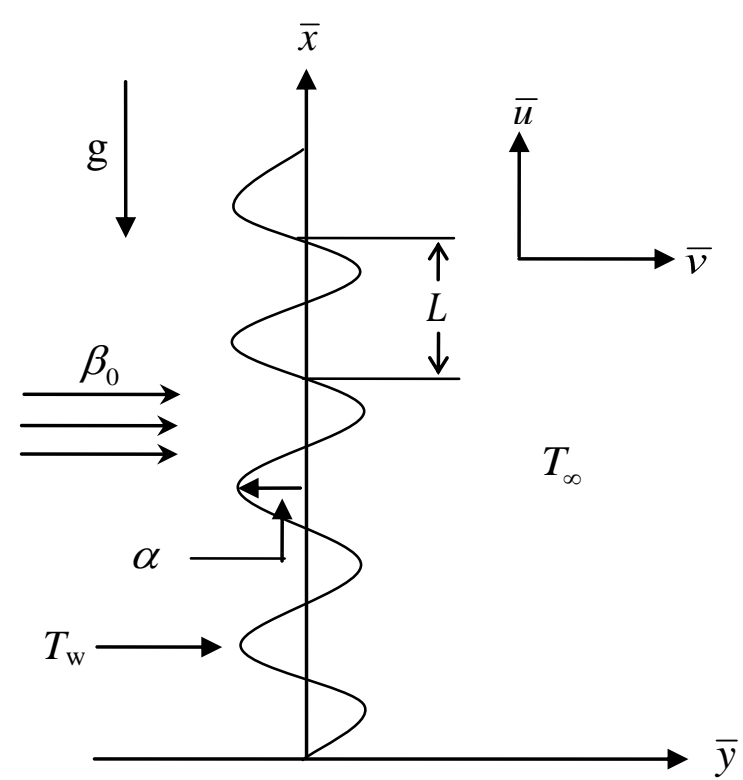

Figure 1. The coordinate system and the physical model

The governing equations of such flow of magnetic field in presence of heat generation/absorption with viscosity variation along a vertical wavy surface under the usual Boussinesq approximations can be written in a dimensional form as:

$\frac{\partial \bar{u}}{\partial \bar{x}}+\frac{\partial \bar{v}}{\partial \bar{y}}=0$ 


$$
\begin{aligned}
& \bar{u} \frac{\partial \bar{u}}{\partial \bar{x}}+\bar{v} \frac{\partial \bar{u}}{\partial \bar{y}}=-\frac{1}{\rho} \frac{\partial \bar{p}}{\partial \bar{x}}+\frac{1}{\rho} \nabla \cdot(\mu \nabla \bar{u}) \\
& +g \beta\left(T-T_{\infty}\right)-\frac{\sigma_{0} \beta_{0}^{2}}{\rho} \bar{u} \\
& \bar{u} \frac{\partial \bar{v}}{\partial \bar{x}}+\bar{v} \frac{\partial \bar{v}}{\partial \bar{y}}=-\frac{1}{\rho} \frac{\partial \bar{p}}{\partial \bar{y}}+\frac{1}{\rho} \nabla \cdot(\mu \nabla \bar{v}) \\
& \bar{u} \frac{\partial T}{\partial \bar{x}}+\bar{v} \frac{\partial T}{\partial \bar{y}}=\frac{k}{\rho C_{p}} \nabla^{2} T+\frac{Q_{0}}{\rho C_{p}}\left(T-T_{\infty}\right)
\end{aligned}
$$

where $(\bar{x}, \bar{y})$ are the dimensional coordinates along and normal to the tangent of the surface and $(\bar{u}, \bar{v})$ are the velocity components parallel to $(\bar{x}, \bar{y})$, $\nabla^{2}\left(=\partial^{2} / \partial x^{2}+\partial^{2} / \partial y^{2}\right)$ is the Laplacian operator, $g$ is the acceleration due to gravity, $\bar{p}$ is the dimensional pressure of the fluid, $\rho$ is the density, $\beta_{0}$ is the strength of magnetic field, $\sigma_{0}$ is the electrical conduction, $k$ is the thermal conductivity of the fluid, $\beta$ is the coefficient of thermal expansion, $\mu(T)$ is the dynamic viscosity of the fluid in the boundary layer region depending on the fluid temperature, $v(=\mu / \rho)$ is the kinematics viscosity and $C_{p}$ is the specific heat due to constant pressure. The amount of heat generated or absorbed per unit volume is $Q_{0}\left(T-T_{\infty}\right)$, $Q_{0}$ being a constant, which may take either positive or negative values. The source term represents the heat generation when $Q_{0}>0$ and the heat absorption when $Q_{0}<0$.

The boundary conditions relevant to the above problem are

$$
\begin{aligned}
& \bar{u}=0, \bar{v}=0, T=T_{w} \quad \text { at } \bar{y}=\bar{y}_{w}=\bar{\sigma}(\bar{x}) \\
& \bar{u}=0, \quad T=T_{\infty}, \quad \bar{p}=p_{\infty} \quad \text { as } \quad \bar{y} \rightarrow \infty
\end{aligned}
$$

where $T_{w}$ is the surface temperature, $T_{\infty}$ is the ambient temperature of the fluid and $p_{\infty}$ is the pressure of fluid outside the boundary layer.

The variable viscosity chosen in this study which is introduced by Charraudeau [1] and used by Hossain et al. [12] as follows:

$$
\mu=\mu_{\infty}\left[1+\varepsilon^{*}\left(T-T_{\infty}\right)\right]
$$

where $\mu_{\infty}$ is the viscosity of the ambient fluid and $\varepsilon^{*}$ is a constant evaluated at the film temperature of the flow $T_{f}=1 / 2\left(T_{w}+T_{\infty}\right)$.

Following Yao [2], we now introduce the following nondimensional variables:

$$
\begin{aligned}
& x=\frac{\bar{x}}{L}, \quad y=\frac{\bar{y}-\bar{\sigma}}{L} G r^{\frac{1}{4}}, p=\frac{L^{2}}{\rho v^{2}} G r^{-1} \bar{p} \\
& u=\frac{\rho L}{\mu_{\infty}} G r^{-1 / 2} \bar{u}, v=\frac{\rho L}{\mu_{\infty}} G r^{-1 / 4}\left(\bar{v}-\sigma_{x} \bar{u}\right) \\
& \sigma_{x}=\frac{d \bar{\sigma}}{d \bar{x}}=\frac{d \sigma}{d x}, G r=\frac{g \beta\left(T_{w}-T_{\infty}\right)}{v^{2}} L^{3}, \theta=\frac{T-T_{\infty}}{T_{w}-T_{\infty}}
\end{aligned}
$$

where $\theta$ is the dimensionless temperature function and $v(=\mu / \rho)$ is the kinematic viscosity. Introducing the above dimensionless dependent and independent variables into Eqs. (2)-(5), the following dimensionless form of the governing equations are obtained after ignoring terms of smaller orders of magnitude in $G r$, the Grashof number defined in (8).

$$
\begin{aligned}
& \frac{\partial u}{\partial x}+\frac{\partial v}{\partial y}=0 \\
& u \frac{\partial u}{\partial x}+v \frac{\partial u}{\partial y}=-\frac{\partial p}{\partial x}+G r^{1 / 4} \sigma_{x} \frac{\partial p}{\partial y}+\left(1+\sigma_{x}^{2}\right)(1+\varepsilon \theta) \frac{\partial^{2} u}{\partial y^{2}} \\
& +\varepsilon\left(1+\sigma_{x}^{2}\right) \frac{\partial \theta}{\partial y} \frac{\partial u}{\partial y}-M u+\theta \\
& \sigma_{x}\left(u \frac{\partial u}{\partial x}+v \frac{\partial u}{\partial y}\right)=-G r^{1 / 4} \frac{\partial p}{\partial y}+\sigma_{x}\left(1+\sigma_{x}^{2}\right)(1+\partial \theta) \frac{\partial^{2} u}{\partial y^{2}} \\
& +\varepsilon \sigma_{x}\left(1+\sigma_{x}^{2}\right) \frac{\partial \theta}{\partial y} \frac{\partial u}{\partial y}-\sigma_{x x} u^{2} \\
& u \frac{\partial \theta}{\partial x}+v \frac{\partial \theta}{\partial y}=\frac{1}{\operatorname{Pr}}\left(1+\sigma_{x}^{2}\right) \frac{\partial^{2} \theta}{\partial y^{2}}+Q \theta
\end{aligned}
$$

In the above equations $\operatorname{Pr}, Q, \varepsilon$ and $M$ are respectively known as the Prandtl number, heat generation/absorption parameter, viscosity variation parameter and magnetic parameter, which are defined as

$$
\begin{aligned}
& \operatorname{Pr}=\frac{C_{p} \mu_{\infty}}{k}, Q=\frac{Q_{0} L^{2}}{\mu C p G r^{1 / 2}}, \varepsilon=\varepsilon^{*}\left(T_{w}-T_{\infty}\right) \text { and } \\
& M=\frac{\sigma_{0} \beta_{0}^{2} L^{2}}{\mu G r^{1 / 2}}
\end{aligned}
$$

It can easily be seen that the convection induced by the wavy surface is described by Eqs. (9)-(12). We further notice that, Eq. (11) indicates that the pressure gradient along the $y$-direction is $O\left(G r^{-1 / 4}\right)$, which implies that lowest order pressure gradient along $x$-direction can be determined from the inviscid flow solution. For the present problem this pressure gradient $(\partial p / \partial x=0)$ is zero. Eq. (11) further shows that $G r^{1 / 4} \partial p / \partial y$ is $\boldsymbol{O}(1)$ and is determined by the left-hand side of this equation. Thus, the elimination of $\partial p / \partial y$ from Eqs. (10) and (11) leads to

$$
\begin{aligned}
& u \frac{\partial u}{\partial x}+v \frac{\partial u}{\partial y}=\left(1+\sigma_{x}^{2}\right)(1+\varepsilon \theta) \frac{\partial^{2} u}{\partial y^{2}}-\frac{\sigma_{x} \sigma_{x x}}{1+\sigma_{x}^{2}} u^{2} \\
& +\varepsilon\left(1+\sigma_{x}^{2}\right) \frac{\partial u}{\partial y} \frac{\partial \theta}{\partial y}-\frac{M}{1+\sigma_{x}^{2}} u+\frac{1}{1+\sigma_{x}^{2}} \theta
\end{aligned}
$$

The corresponding boundary conditions for the present problem then turn into

$$
\left.\begin{array}{l}
u=v=0, \quad \theta=1 \quad \text { at } \quad y=0 \\
u=\theta=0, \quad p=0 \quad \text { as } \quad y \rightarrow \infty
\end{array}\right\}
$$

Now we introduce the following transformations to reduce the governing equations to a convenient form: 
$\psi=x^{3 / 4} f(x, \eta), \quad \eta=y x^{-1 / 4}, \quad \theta=\theta(x, \eta)$

where $f(\eta)$ is the dimensionless stream function, $\eta$ is the pseudo similarity variable and $\psi$ is the stream function that satisfies the continuity equation and is related to the velocity components in the usual way as

$$
u=\frac{\partial \psi}{\partial y}, \quad v=-\frac{\partial \psi}{\partial x}
$$

Introducing the transformations given in Eq. (15) into Eqs. (13) and (12) are transformed into the new co-ordinate system. Thus the resulting equations are

$$
\begin{aligned}
& \left(1+\sigma_{x}^{2}\right)(1+\varepsilon \theta) f^{\prime \prime \prime}+\frac{3}{4} f f^{\prime \prime}-\left(\frac{1}{2}+\frac{x \sigma_{x} \sigma_{x x}}{1+\sigma_{x}^{2}}\right) f^{\prime 2}+\frac{1}{1+\sigma_{x}^{2}} \theta \\
& -\frac{M x^{1 / 2}}{1+\sigma_{x}^{2}} f^{\prime}+\varepsilon\left(1+\sigma_{x}^{2}\right) \theta^{\prime} f^{\prime \prime}=x\left(f^{\prime} \frac{\partial f^{\prime}}{\partial x}-f^{\prime \prime} \frac{\partial f}{\partial x}\right) \\
& \frac{1}{\operatorname{Pr}}\left(1+\sigma_{x}^{2}\right) \theta^{\prime \prime}+\frac{3}{4} f \theta^{\prime}+x^{1 / 2} Q \theta=x\left(f^{\prime} \frac{\partial \theta}{\partial x}-\theta^{\prime} \frac{\partial f}{\partial x}\right)
\end{aligned}
$$

The boundary conditions as mentioned in Eq. (14) then take the form given below:

$$
\begin{aligned}
& f(x, o)=f^{\prime}(x, o)=0, \quad \theta(x, o)=1 \\
& f^{\prime}(x, \infty)=0, \quad \theta(x, \infty)=0
\end{aligned}
$$

\section{LOCAL SKIN FRICTION COEFFICIENT AND THE LOCAL RATE OF HEAT TRANSFER}

The quantities of physical interested, namely, the shearing stress $\tau_{\mathrm{w}}$ in terms of the skin friction coefficient $C_{f x}$ and the rate of heat transfer in terms of Nusselt number $N u_{x}$ are prescribed by:

$$
C_{f x}=\frac{2 \tau_{w}}{\rho U_{\infty}^{2}} \text { and } N u_{x}=\frac{q_{w} x}{k\left(T_{w}-T_{\infty}\right)}
$$

where $\tau_{w}=(\mu \bar{n} . \nabla \bar{u})_{y=0}$ and $q_{w}=-k(\bar{n} . \nabla T)_{y=0}$

Using the transformations (15) and Eq. (21) into Eq. (20), the local skin friction coefficient $C_{f x}$ and the rate of heat transfer in terms of the local Nusselt number $N u_{x}$ takes the following form:

$$
\begin{aligned}
& C_{f x}(G r / x)^{1 / 4} / 2=(1+\varepsilon) \sqrt{1+\sigma_{x}^{2}} f^{\prime \prime}(x, o) \\
& N u_{x}(G r / x)^{-1 / 4}=-\sqrt{1+\sigma_{x}^{2}} \theta^{\prime}(x, o)
\end{aligned}
$$

\section{METHOD OF SOLUTION}

The transformed boundary layer equations solved numerically with the help of implicit finite difference method together with the Keller-Box scheme [18]. To begin with the partial differential equations are first converted into a system of first order differential equations. Then these equations are expressed in finite difference forms by approximating the functions and their derivatives in terms of the center differences. Denoting the mesh points in the $x$ and $\eta$ plane by $x_{i}$ and $\eta_{j}$ where $i=1,2, \ldots, M$ and $j=1$, $2, \ldots, N$, central difference approximations are made, such that those equations involving $x$ explicitly are centered at $\left(x_{i-1 / 2}, \eta_{j-1 / 2}\right)$ and the remainder at $\left(x_{i,} \eta_{j-1 / 2}\right)$, where $\eta_{j-1 / 2}=1 / 2\left(\eta_{j}+\eta_{j-1}\right)$ etc. The above central difference approximations reduce the system of first order differential equations to a set of non-linear difference equations for the unknown at $x_{i}$ in terms of their values at $x_{i-1}$. The resulting set of non-linear difference equations are solved by using the Newton's quasi-linearization method. The Jacobian matrix has a block-tridiagonal structure and the difference equations are efficiently solved using a block-matrix version of the Thomas algorithm. In the program test, a finer axial step size is tried and find to give acceptable accuracy. A uniform grid of 201 points is used in $x$-direction with $\Delta x=0.05$, while a non-uniform grid of 76 points lying between $\eta=0.0$ and 10.017 is chosen. Grid points are concentrated towards the heated surface in order to improve resolution and the accuracy of the computed values of the surface shear stress and rate of heat transfer. During the program test, the convergent criteria for the relative errors between two iterations are less $10^{-}$ 5 . It means that iterative procedure is stopped when the maximum change between successive iterates is less then $10^{-5}$.

\section{RESULTS AND DISCUSSION}

The problem of MHD natural convection flow of a viscous incompressible fluid in presence of heat generation/absorption with variable viscosity along a vertical wavy surface with uniform surface temperature has been investigated. In this section, numerical results of the skin friction coefficient $C_{f x}$, the rate of heat transfer in terms of the Nusselt number $N u_{x}$, the streamlines and the isotherms are obtained for various values of the viscosity parameter ranging from $\varepsilon=0.0$ (constant viscosity) to 15.0 , heat generation parameter $Q=0.0$ to 1.0 and heat absorption parameter $Q=0.0$ to -0.6 while magnetic parameter $M=0.5$, the amplitude-to-length ratio of the wavy surface $\alpha=0.3$ and $\operatorname{Pr}=0.73$ which correspond to the air at $2100^{\circ} \mathrm{K}$ are fixed.

The analysis of the effect of heat generation/absorption parameter $Q$ on the local skin friction coefficient $C_{f x}$ and the rate of heat transfer in terms of the local Nusselt number $N u_{x}$ against $x$ from the wavy surface while $\alpha=0.3, M=0.5, \varepsilon=5.0$ and $\operatorname{Pr}=0.73$ are illustrated in Figs. 2 and 3 respectively. From Fig. 2, it is observed that the skin friction coefficient significant increases along the upstream direction of the surface and the rate of heat transfer from the heated surface decreases with the increase of the heat generation parameter $Q$. Heat generation mechanism creates a layer of hot fluid near the surface and finally the resultant temperature of the fluid exceeds the surface temperature. For this reason the rate of heat transfer in terms of the local Nusselt number from the surface decreases. Owing to the enhanced temperature, the viscosity of the fluid increases and the corresponding local skin friction coefficient increases. On the other hand, the presence of heat absorption $(Q<0)$ creates a layer of cold 
fluid adjacent to the heated surface and therefore the rate of heat transfer in terms of the local Nusselt number from the surface increases. The skin friction coefficient decreases along the downstream direction of the surface in this case. The amplitude of the skin friction coefficient for the heat generation case is strongly higher than that of the skin friction coefficient for heat absorption case.

The skin friction coefficient $C_{f x}$ and local rate of heat transfer $N u_{x}$ for influence of viscosity parameter $\varepsilon=$ $(0.0,4.0,8.0,10.0$ and 15.0) for heat generation case $Q=0.4, M=0.5, \alpha=0.3$ and $\operatorname{Pr}=0.73$ are displayed by Figs. 4(a) and 4(b). It is noted that an increase in the value of variable viscosity variation parameter $\varepsilon$, the skin friction coefficient increases monotonically along the upstream direction of the surface and to decrease of the heat transfer rates. Here it is concluded that for high viscous fluid the skin friction is higher and the corresponding rate of heat transfer is slow. The maximum values of local skin friction coefficient $C_{f x}$ are 0.96896 and 23.13310 for $\varepsilon=0.0$ and 15.0 respectively which occur at the different values of $x$. Finally it is seen that the local skin friction coefficient increases by approximately $96 \%$ as $\varepsilon$ changes from 0.0 to 15.0 .
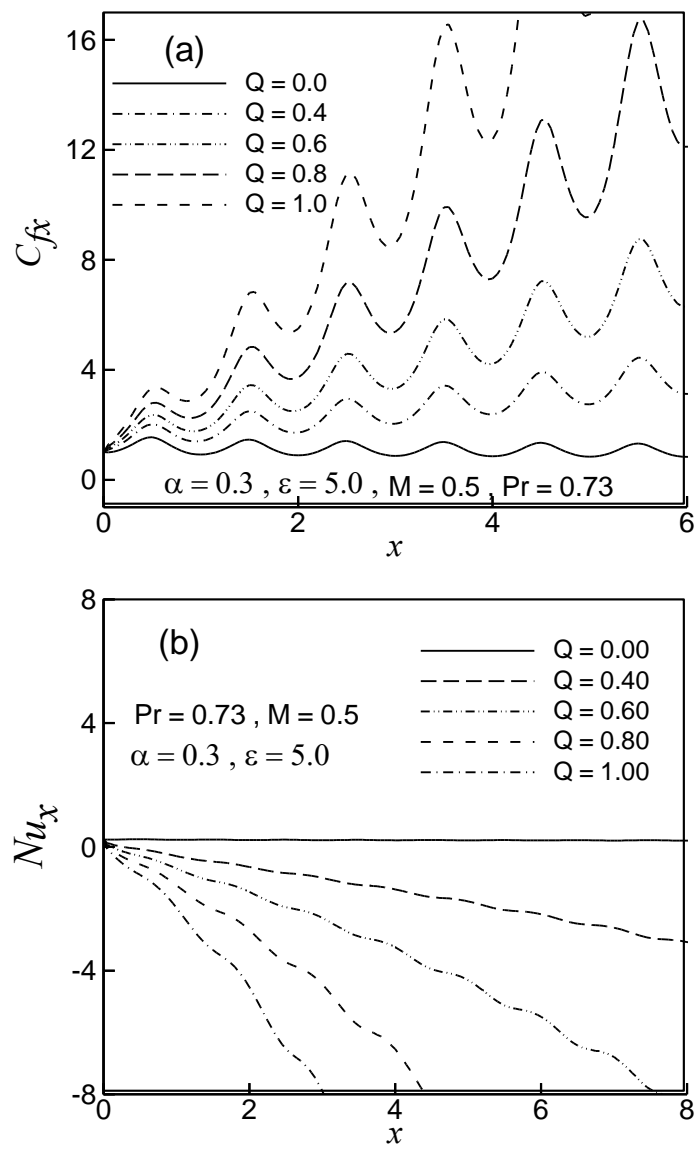

Figure 2. Variation of (a) skin friction coefficient $C_{f x}$ and (b) rate of heat transfer $N u_{x}$ against $x$ for different values of heat generation parameter $Q$ while $\operatorname{Pr}=$ $0.73, \alpha=0.3, M=0.5$ and $\varepsilon=5.0$.
The influence of heat generation/absorption parameter $Q$ on the formation of streamlines which are plotted for $\alpha=0.3, M=0.5, \varepsilon=5.0$ and $\operatorname{Pr}=0.73$ are depicted by the Fig. 5. It is noted that, in the case of heat absorption the maximum value of $\psi$, that is, $\psi_{\max }$ is 1.37 for $Q=-0.4$ and for heat generation case the maximum values of $\psi$, that is, $\psi_{\max }$ is 15.28 for $Q$ $=0.4$ and for $Q=1.0$ the value of $\psi_{\max }$ is 26.64 . It is concluded that the velocity distribution of the fluid flow for the case of heat generation is higher than that of the heat absorption case.

Fig. 6 deals with the effect of heat generation/absorption parameter $Q$ on the isotherms profile which are plotted for $\alpha=0.3, M=0.5, \varepsilon=5.0$ and $\operatorname{Pr}=0.73$. In the presence of heat generation effect $(Q>0)$, the thermal state of the fluid increases, causing the thermal boundary layer becomes thicker. On the other hand, an opposite situation is observed on the temperature field within the boundary layer in the case of heat absorption.
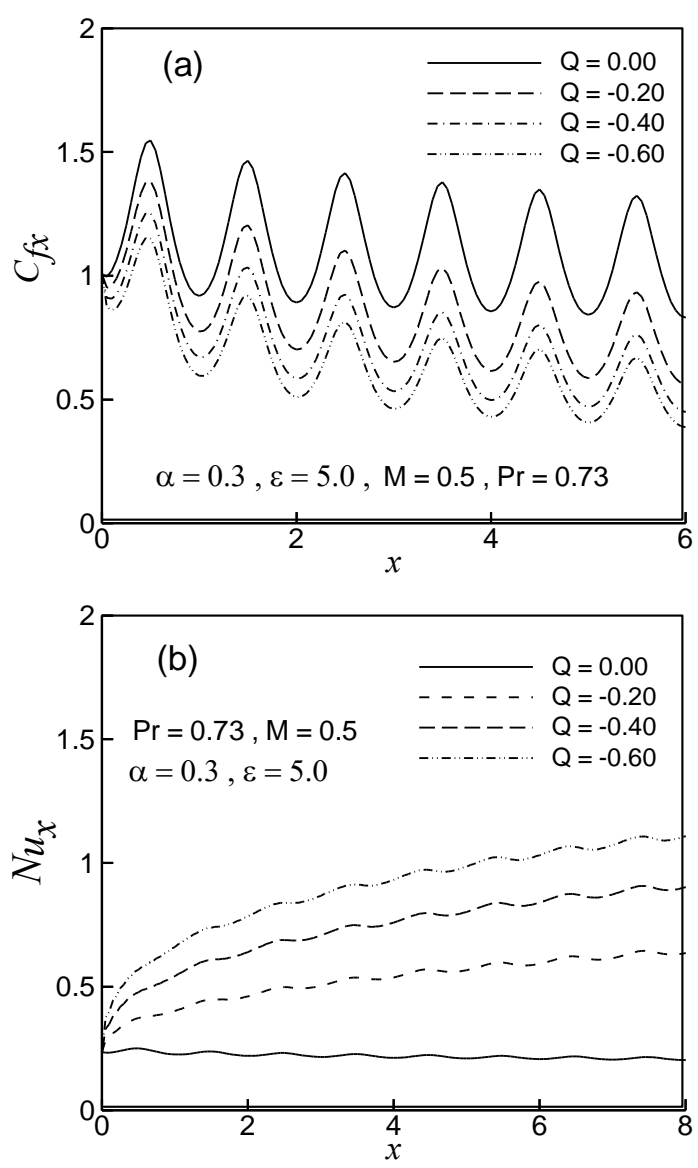

Figure 3. Variation of (a) skin friction coefficient $C_{f x}$ and (b) rate of heat transfer $N u_{x}$ against $x$ for different values of heat absorption parameter $\mathrm{Q}$ while $\mathrm{Pr}=$ $0.73, \alpha=0.3, M=0.5$ and $\varepsilon=5.0$. 
Figs. 7 and 8 illustrate the effect of viscosity parameter $\varepsilon$ on the development of streamlines and isotherms profile which are plotted for the amplitudeto-length ratio of the wavy surface $\alpha=0.3$, Prandtl number $\operatorname{Pr}=0.73, M=0.5$ and $Q>0(Q=0.4)$. From Fig. 7, it is seen that owing to the effect of variable viscosity, the flow rate in the boundary layer decreases. The maximum values of $\psi$, that is, $\psi_{\max }$ are 15.24 and 14.44 for viscosity parameter $\varepsilon=0.0$ and 15.0 respectively. From Fig. 8, it is observed that temperature dependent viscosity affect the isotherms and leads to thicker the thermal boundary layer. Finally it is concluded that the effect of viscosity parameter the velocity of the fluid flow decreases and temperature distribution within the boundary layer increases.
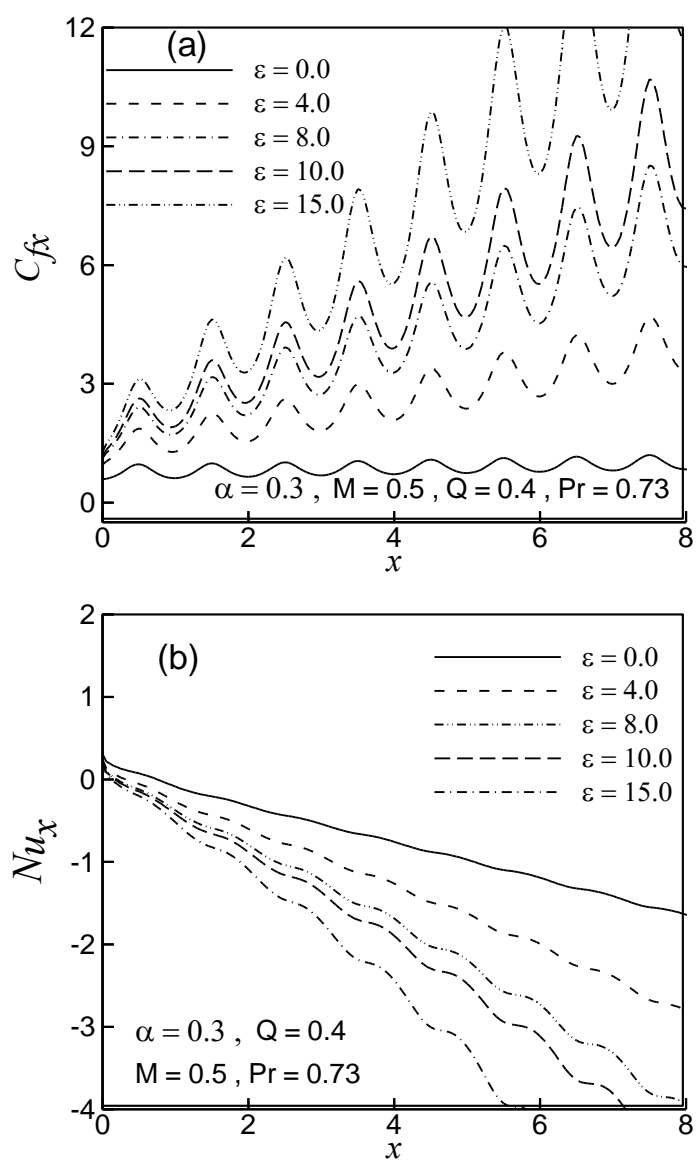

Figure 4. Variation of (a) skin friction coefficient $C_{f x}$ and (b) rate of heat transfer $N u_{x}$ against $x$ for different values of $\varepsilon$ while $\alpha=0.3, M=0.5, Q=0.4$ and $\operatorname{Pr}=$ 0.73 .
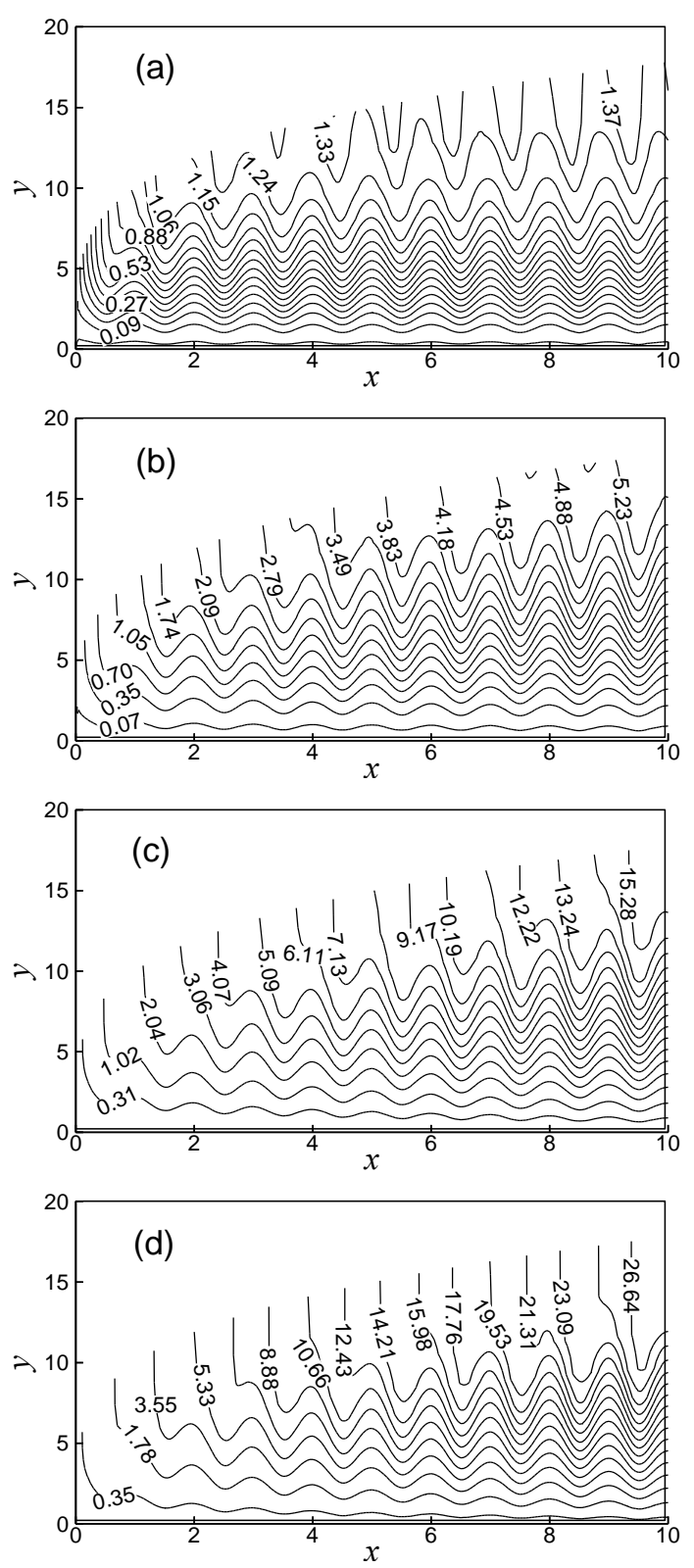

Figure 5. Streamlines for (a) $Q=-0.4$ (b) $Q=0.0$ (c) $Q=0.40$ (d) $Q=1.0$ while $\operatorname{Pr}=0.73, M=0.5, \varepsilon=$ 5.0 and $\alpha=0.3$.

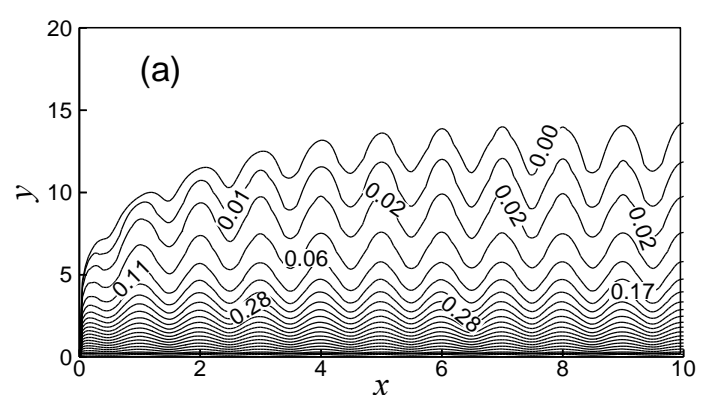



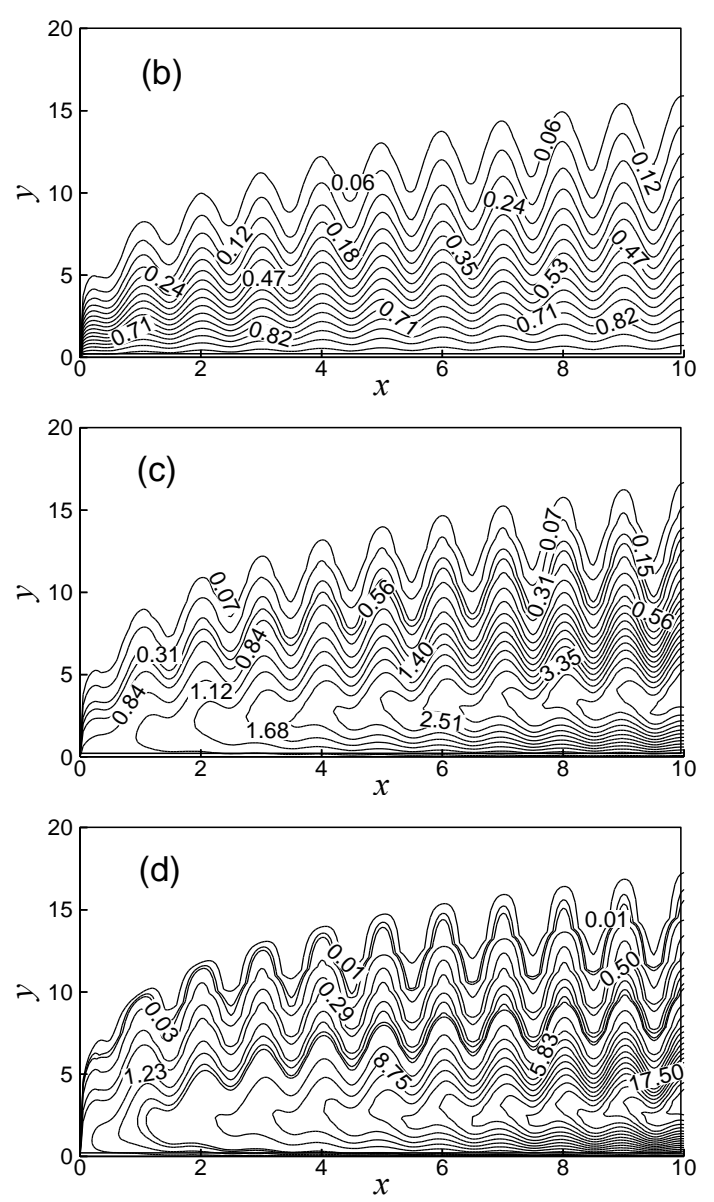

Figure 6. Isotherms for (a) $Q=-0.4$ (b) $Q=0.0$ (c) $Q$ $=0.40$ (d) $Q=1.0$ while $\operatorname{Pr}=0.73, M=0.5, \varepsilon=5.0$ and $\alpha=0.3$.
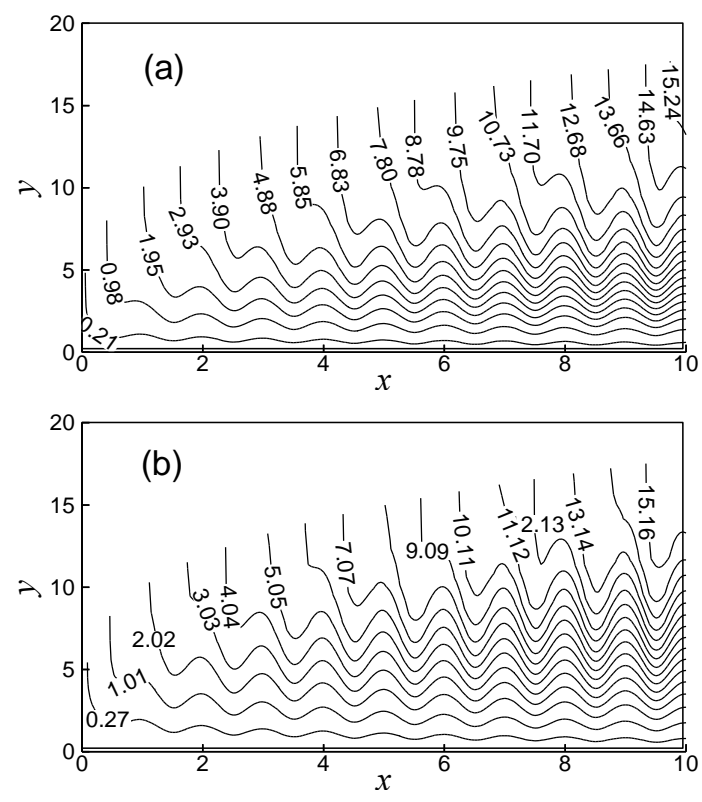
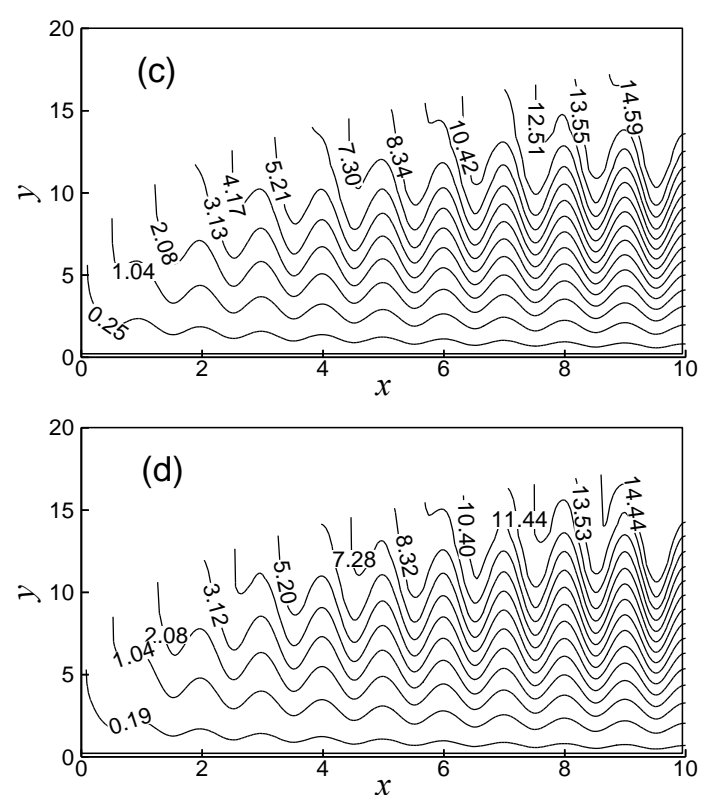

Figure 7. Streamlines for (a) $\varepsilon=0.0$ (b) $\varepsilon=4.0$ (c) $\varepsilon$ $=10.0$ (d) $\varepsilon=15.0$ while $\operatorname{Pr}=0.73, M=0.5, Q=0.4$ and $\alpha=0.3$.
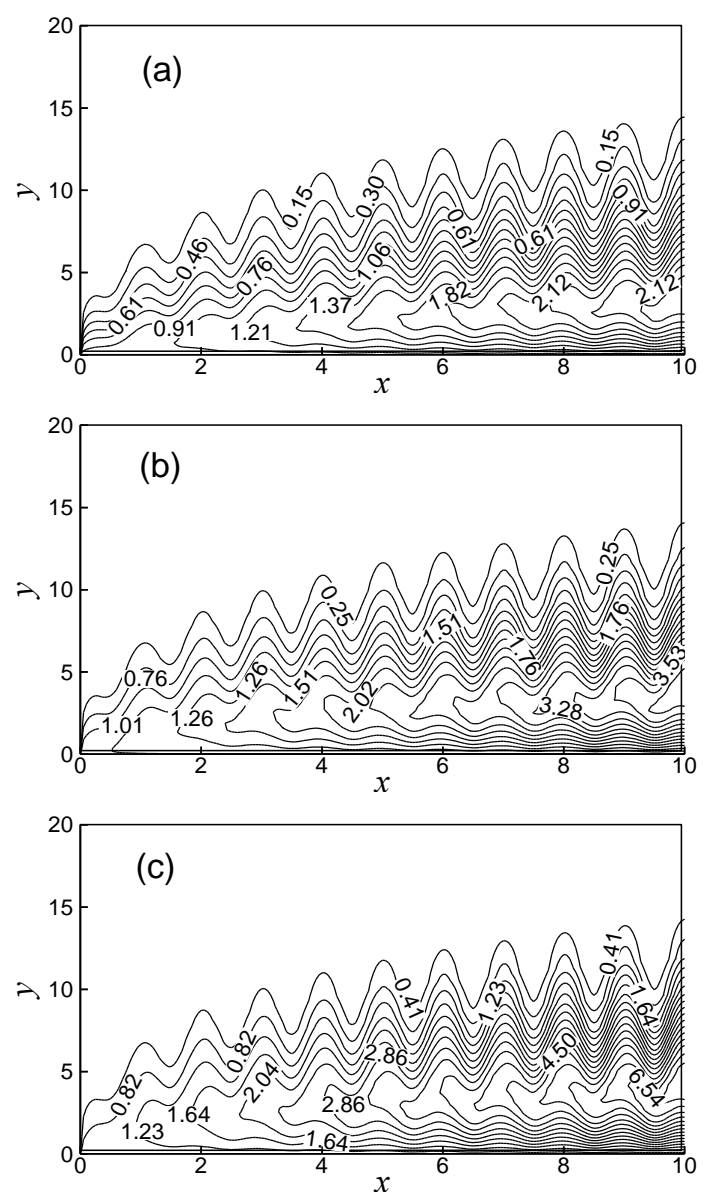


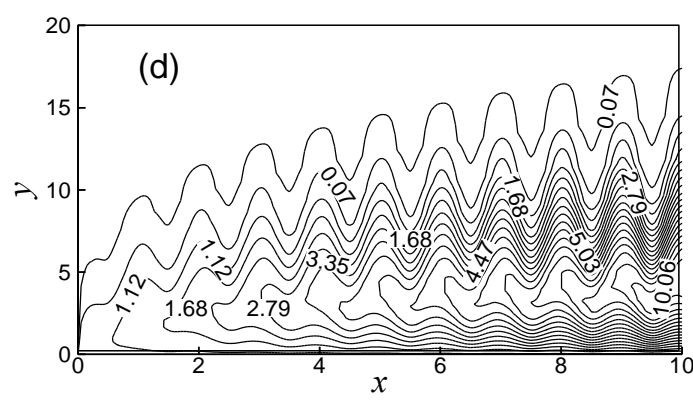

Figure 8. Isotherms for (a) $\varepsilon=0.0$ (b) $\varepsilon=4.0$ (c) $\varepsilon=$ 10.0 (d) $\varepsilon=15.0$ while $\operatorname{Pr}=0.73, M=0.5, Q=0.4$ and $\alpha=0.3$.

\section{COMPARISON WITH PREVIOUS WORK AND PROGRAM VALIDATION}

A comparison of the present numerical results of the skin friction coefficient, $f^{\prime \prime}(x, 0)$ and the heat transfer, $-\theta^{\prime}(x, 0)$ with the results obtained by Hossain et al. [12] are depicted in Table 1. Here, the magnetic parameter $M$, viscosity variation parameter $\varepsilon$ and heat generation/absorption parameter $Q$ are ignored with different values of Prandtl number $\operatorname{Pr}=(1.0,10,25.0$ and 100.0) are chosen. Excellent agreement is achieved with the present results and the numerical results of Hossain et al. [12]. These validations boost the confidence in the numerical code to carry on with the above stated objectives of the current investigation.

Table 1: Comparison of the present numerical results of skin friction coefficient, $f^{\prime \prime}(x, 0)$ and the heat transfer, $-\theta^{\prime}(x, 0)$ with Hossain et al. [12] for the variation of Prandtl number Pr while $M=0.0, \varepsilon=$ $0.0, Q=0.0$ with $\alpha=0.1$.

\begin{tabular}{|c|c|c|c|c|}
\hline \multirow{2}{*}{$\operatorname{Pr}$} & \multicolumn{2}{|c|}{$f^{\prime \prime}(x, 0)$} & \multicolumn{2}{c|}{$-\theta^{\prime}(x, 0)$} \\
\cline { 2 - 5 } & $\begin{array}{c}\text { Hossain } \\
\text { et al. } \\
{[12]}\end{array}$ & $\begin{array}{c}\text { Present } \\
\text { work }\end{array}$ & $\begin{array}{c}\text { Hossain } \\
\text { et al. } \\
{[12]}\end{array}$ & $\begin{array}{c}\text { Present } \\
\text { work }\end{array}$ \\
\hline 1.0 & 0.908 & 0.91084 & 0.401 & 0.39914 \\
\hline 10.0 & 0.591 & 0.59482 & 0.825 & 0.82315 \\
\hline 25.0 & 0.485 & 0.48910 & 1.066 & 1.06405 \\
\hline 50.0 & 0.485 & 0.41880 & 1.066 & 1.28351 \\
\hline 100 & 0.352 & 0.35690 & 1.542 & 1.54198 \\
\hline
\end{tabular}

\section{CONCLUSION}

The effect of heat generation/absorption on MHD natural convection flow with temperature dependent viscosity along a uniformly heated vertical wavy surface has been analyzed. The effects of pertinent parameters on flow and heat transfer characteristics are discussed in detail. The results of the numerical analysis lead to the following conclusions:

- The skin friction coefficient increases noticeably and the rate of heat transfer decreases in the case of heat generation. For this case, the buoyancy force increases that increase the flow rate in the boundary layer. For the heat absorption case, the opposite phenomenon occurs when the axial distance variable $x$ increases.

- The velocity and thermal boundary layer grow thick substantially for the effect of heat generation. But the opposite results obtained in the case of heat absorption.

- Increasing values of temperature dependent viscosity variation parameter $\varepsilon$, the skin friction coefficient significantly increases and the local rate of heat transfer decreases in the downstream region.

- The velocity of the fluid flow decreases and the temperature distribution of the fluid flow within the boundary layer significantly increase for increasing values of $\varepsilon$.

\section{REFERENCES}

[1] J. Charraudeau, "Influence de gradients de properties physiques en convection force application au cas du tube", Int. J. Heat Mass Transfer, Vol. 18, pp. 87-95, 1975.

[2] L. S. Yao, "Natural convection along a vertical wavy surface", ASME J. Heat Transfer, Vol. 105, pp. 465-468, 1983.

[3] L. S. Yao, “A note on Prandtl's transposition theorem", ASME J. Heat Transfer, Vol. 110, pp. 503-507, 1988.

[4] K. N. Mehta and S. Sood, "Transient free convection flow with temperature dependent viscosity in a fluid saturated porous media", Int. J. Engrg. Sci., Vol. 30, pp. 1083-1087, 1992.

[5] S. G. Moulic and L. S. Yao, "Natural convection along a wavy surface with uniform heat flux", ASME J. Heat Transfer, Vol. 111, pp. 1106-1108, 1989.

[6] K. Vejravelu and A. Hadjinicolaou, "Heat transfer in a viscous fluid over a stretching sheet with viscous dissipation and internal heat generation", Int. comm. Heat Transfer, Vol. 20, pp. 417-430, 1993.

[7] N. G. Kafoussius and E. M. Williams, "The effect of temperature dependent viscosity on the free convective laminar boundary layer flow past a vertical isothermal flat plate", Acta Mechanica, Vol. 110, pp. 123-137, 1995.

[8] K. C. A. Alam, M. A. Hossain and D. A. S. Rees, "Magnetohydrodynamic free convection along a vertical wavy surface", Int. J. Appl. Mech. Engrg, Vol. 1, pp. 555-566, 1997.

[9] M. A. Hossain and D. A. S. Rees, "Combined heat and mass transfer in natural convection flow from a vertical wavy surface", Acta Mechanica, Vol. 136, pp. 133-141, 1999.

[10] M. A. Hossain, M. S. Munir and D. A. S. Rees, "Flow of viscous incompressible fluid with temperature dependent viscosity and thermal 
conductivity past a permeable wedge with uniform surface heat flux", Int. J. Therm. Sci., Vol. 39, pp. 635-644, 2000.

[11] C. Y. Cheng, "Natural convection heat and mass transfer near a vertical wavy surface with constant wall temperature and concentration in a porous medium", Int. Comm. Heat Mass Transfer, Vol. 27, pp. 1143-1154, 2000.

[12] M. A. Hossain, S. Kabir and D. A. S. Rees, "Natural convection of fluid with temperature dependent viscosity from heated vertical wavy surface", Z. Angew. Math. Phys., Vol. 53, pp. 48-52, 2002.

[13] M. M. Molla, M. A. Hossain and L. S. Yao, "Natural convection flow along a vertical wavy surface with uniform surface temperature in presence of heat generation/absorption", Int. J. Therm. Sci., Vol. 43, pp. 157-163, 2004.

[14] A. A. Mamun, Z. R. Chowdhury, M. A. Azim and M. M. Molla, "MHD-conjugate heat transfer analysis for a vertical flat plate in presence of viscous dissipation and heat generation", Int. comm. Heat Transfer, Vol. 35, pp. 1275-1280, 2008.
[15] M. M. Molla, M. A. Hossain and R. R. S. Gorla, "Natural convection laminar flow with temperature dependent viscosity and thermal conductivity along a vertical wavy surface", Int. J. Fluid Mech. Research, Vol. 36, pp.272-288, 2009.

[16] G. Palani and K.-Y. Kim, "Numerical study on a vertical plate with variable viscosity and thermal conductivity", Arch Appl. Mech., Vol. 80, pp. 711725, 2010.

[17] N. Parveen and M. A. Alim, "Effect of temperature-dependent variable viscosity on magnetohydrodynamic natural convection flow along a vertical wavy surface", International Scholarly Research Network Mechanical Engineering, Vol. 2011, Article ID 505673, pp. 1-10, 2011.

[18] H. B. Keller, "Numerical methods in boundary layer theory”, Ann. Rev. Fluid Mech., Vol. 10, pp. 417-433, 1978

[19] T. Cebeci and P. Bradshaw, "Physical and computational aspects of convective heat transfer", Springer, New York, 1984. 